\title{
Antropolog
}

\section{Relación entre el estilo de vida de una joven deportista de alto rendimiento y los patrones funcionales de salud de Marjory Gordon}

\section{Relationship between the lifestyle of a young sportsman high performance and functional health patterns Marjory Gordon}

\section{Relação entre o estilo de vida de um jovem esportista de alto desempenho e padrões funcionais de saúde Marjory Gordon}

\author{
Juan Manuel Fabra Heredia ${ }^{1}$ Lina Casadó Marín \\ ${ }^{1}$ Estudiante de Grado en Enfermería en URV; juanrodiles@hotmail.com; 636308160) \\ ${ }^{1}$ Estudiante de Grado en Enfermería en URV (Universidad Rovira iVirgili). \\ ${ }^{2}$ Doctora en Antropología, Departamento de enfermería de la URV(Universidad Rovira iVirgili).
}

Cómo citar este relato en edición digital: Fabra Heredia, J.M. y Casadó Marín, L. (2014) Relación entre el estilo de vida de una joven deportista de alto rendimiento y los patrones funcionales de salud de Marjory Gordon. Cultura de los Cuidados (Edición digital) 18, 38. Disponible en: http://dx.doi.org/10.7184/cuid.2014.38.13>

Correspondencia: Correo electónico: juanrodiles@hotmail.com Recibido: 20/11/2013; Aceptado: 11/01/2014

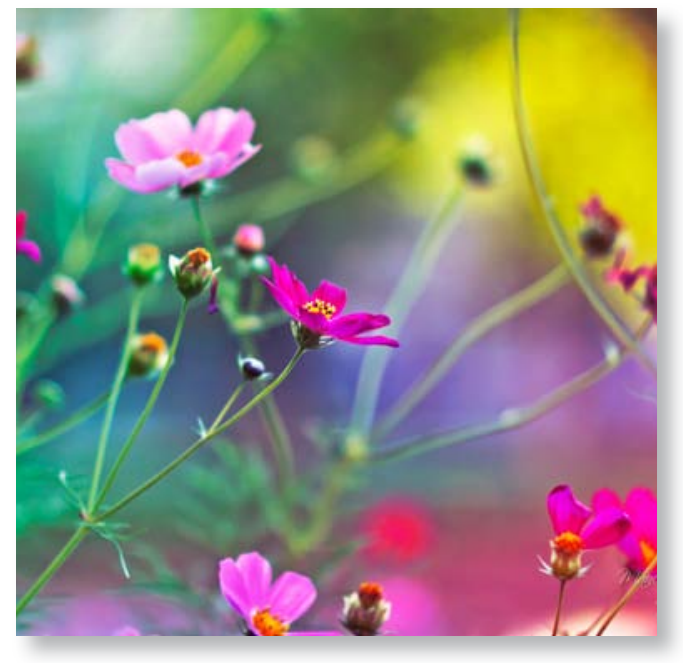

ABSTRACT

This case study explores the benefits of Marjory Gordon's health functional patterns in a high performance young athlete lifestyle. Starting from a holistic perspective and through a nursing functional health care plan in practice, I would like to set the basis for nursing care to high performance athletes.

The results showed that there are some protective factors connected with healthy life patterns as: balanced diet, physical activity and drug use prevention. Moreover, there are some risky behaviour related with the effort and the high dedication in daily life.

Keywords: High performance sport, Functional patterns, Lifestyle, Nursing process evaluation.

\section{RESUMO}

Estudo de caso que busca conhecer e aprender a relação que há entre o estilo de vida de um jovem esportista de alto rendimento e os padrões funcionais da saúde, através da avaliação realizada em um jovem atleta de alto desempenho, desde una perspectiva holística, para adentrar-se nas peculiaridades específicas 
deste estilo de vida e criar um ponto de partida com os cuidados da enfermagem dirigidos a esportistas de alto rendimento.

Em este caso, observa-se fatores protetores relacionados com a tendência de hábitos de vida saudáveis (alimentação, atividade física, não consumo de tóxicos) e condutas de risco derivadas do esforço, tempo e dedicação que requer o esporte de alto nível.

Palavras-chave: Esporte de alto rendimento, Padrões funcionais, Estilo de vida, Avaliação de enfermagem.

\section{RESUMEN}

Estudio de caso que busca conocer y comprender la relación que hay entre el estilo de vida de una joven deportista de alto rendimiento y los patrones funcionales de salud, a través de la valoración realizada a una joven deportista de alto rendimiento, desde una perspectiva holística, para adentrarse en las peculiaridades propias de este estilo de vida y crear un punto de partida para los cuidados de enfermería dirigidos a deportistas de alto rendimiento.

En este caso, se observaron factores protectores relacionados con la tendencia a los hábitos de vida saludables (alimentación, actividad física, no consumo de tóxicos) y conductas de riesgo derivadas del esfuerzo y tiempo de dedicación que requiere el deporte a alto nivel.

Palabras clave: Deporte de alto rendimiento, Patrones funcionales, Estilo de vida. Valoración enfermería.

\section{INTRODUCCIÓN}

Durante los últimos años, se han presentado estudios referentes al deporte de alto rendimiento en los que se relacionaba este estilo de vida con la promoción de la salud o por lo contrario se daba a entender que este tipo de práctica deportiva era un factor de riesgo para algunas dimensiones de la salud de la persona.

Como pequeño repaso de los estudios relacionados con el deporte de alto rendimiento, podemos resaltar, por ejemplo, el trabajo de de Jonathan Ruiz (2010) en el que se revisan quince estudios que asocian de manera inequívoca la participación en competiciones deportivas con la esperanza de vida. Otro defensor del deporte de alto rendimiento es Alejandro Lucía (2006) llegó a la conclusión que el hecho de ser deportista de alto rendimiento, trae consigo un estilo de vida (habitos saludables, dieta, actividad) con un pronóstico muy favorable sobre el riesgo en general de enfermedades y muerte. En la misma linea, Ivana Jürgens (2006) nos muestra un claro aumento de la calidad de vida percibida cuanto mas alto es el grado de implicación en la práctica deportiva. Siendo los deportistas de alto rendimiento los que obtienen el mejor resultado. La otra cara de la moneda, la encontramos, por ejemplo, en el riesgo de lesiones que conlleva el deporte de alto rendimiento, habiendo autores que consideran la práctica deportiva en si en un factor de riesgo (Amato, R. 2000). A su vez, el carácter competitivo de esta rama del deporte, también puede conllevar a una obsesión desencadenante de problemas de autoimagen o trastornos de la conducta alimentaria (Alonso, J. 2006).

Como hemos podido observar hasta ahora la investigación científica sobre los efectos de la práctica deportiva se ha centrado, principalmente, en las repercusiones fisiológicas (De Gracia, M. y Marcó, M. 2000). No obstante, el interés también se ha extendido a la salud mental (Jiménez, M. 2008).

Varios estudios afirman que el ejercicio físico mejora la salud mental. Entre estos be- 
neficios encontramos la reducción de la depresión (Lawlor, D. A. y Hopker, S. W. 2001); la disminución de la reactividad frente al estrés y un aumento de la capacidad de afrontación de este (Holmes, D.S. 1993), y de este modo se reducen los niveles de ansiedad (Akandere, $\mathrm{M}$. y Tekin, A. 2005). ¿Pero pasa lo mismo cuando este deporte, es de alto rendimiento? La principal diferencia del deporte de alto rendimiento frente a la practica de simple actividad física, es el factor competitivo (Muñoz, J. A. 2001).

Los altos valores de competencia percibida en jóvenes deportistas de alto rendimiento, ofrecen altas correlaciones negativas con las dimensiones generadoras de estrés (Arruza, J. A. 2011). Percibidas con frecuencia como causa de amenaza y angustia (Durand, M. 1988) . Pero al mismo tiempo, guardaba relación con las dimensiones positivas recuperadoras del estrés (Arruza, J. A. 2011).

A pesar de esta última afirmación, Sara Márquez (2005) afirma que los deportistas que no afrontan el estrés con las estrategias correctas, son incapaces de interpretar de forma adecuada los eventos relacionados con la actividad deportiva y de reaccionar de manera racional. De este modo experimentan un estrés crónico y prolongado, relacionado con el empeoramiento de la capacidad de ejecución, la aparición de burnout (estado de cansancio mental, emocional y físico generado por una dedicación persistente hacia un objetivo, cuyo logro resulta opuesto a las expectativas de la persona (Freudenberger, H. J. y Richelson, G. 1981)).

Este estrés y tensión debidos al carácter competitivo del deporte de alto rendimiento puede llegar a desencadenar en niños y jóvenes desequilibrios psíquicos (García, M. 1990).

Debido al gran numero de posturas sobre la práctca de ejercicio de alto rendimiento, con este estudio, busco dar una visión global de la vida de un deportista de alto rendimiento y conocer que relación guarda este tipo de práctica deportiva con el estilo de vida de la persona.

Teniendo en cuenta que podemos entender el estilo de vida como la manera de vivir, una serie de actividades, rutinas cotidianas o hábitos, (Guerrero, L. R. 2010); dentro de esa "serie de actividades" se puede enmarcar la practica de deporte de alto rendimiento que podríamos definirlo como una actividad física que obedece normas y sus objetivos son la competencia, el máximo rendimiento y el espectáculo (Ortiz, U. 2001) que se realiza de forma organizada y consciente y está diseñado para aumentar el nivel competitivo y el rendimiento atlético, es un proceso organizado, dirigido, orientado, sistemático, planificado y controlado sobre bases metodológicas.(Gómez, O. 2002). Al igual que al deporte de alto rendimiento, al resto de actividades que construyen el estilo de vida, se denominan hábitos o comportamientos sostenidos, de los cuales se distinguen dos categorías: los que mantienen la salud y promueven la longevidad y aquellos que limitan o resultan dañinos y reducen la esperanza de vida. Estos comportamientos se conocen como factores protectores y conductas de riesgo. (Jenkins, D. 2005; Barrios, H. A. 2007).

Para comprender mejor si el deporte de alto rendimiento dentro de un estilo de vida actúa como factor protector o conducta de riesgo, he realizado una valoración de los patrones funcionales de M. Gordon.

\section{¿Por qué el modelo de M. Gordon y no otra escala de valoración?}

Este modelo de enfermería, ha sido escogido entre otros, porque aporta libertad a la hora de poder realizar preguntas encaminadas hacia un conocimiento concreto, en este caso, la 
relación entre el deporte de alto rendimiento y cada patrón funcional de salud, y ofrece la capacidad de investigar abiertamente sobre cada "comportamiento" o patrón.

Esto se debe a que el modelo de valoración de Gordon, expone sus patrones funcionales cómo "comportamientos más o menos comunes a todas las personas" que contribuyen a su salud (Álvarez, J. 2010) a diferencia del modelo de Virginia Henderson, que nos habla de "necesidades básicas que la persona debe satisfacer" para mantener su integridad y promover su crecimiento (García, A., 2004). Y esto se observa en la propia redacción de las necesidades y patrones, mientras que las necesidades de Henderson dan pié a una respuesta de si o no, los patrones de Gordon hacen cuestionarse el cómo y esto ofrece la libertad de investigación comentada con anterioridad. Por lo tanto, el modelo de Gordon, al no hablar de necesidad, nos ofrece un marco de trabajo más amplio que contempla la variabilidad que puede hallarse entre los diferentes estilos de vida de la población, incluyendo el de deportista de alto rendimiento.

Otro aspecto importante que me ha hecho decantarme por el modelo de Gordon, frente a otros cómo el de Henderson, es el hecho de que Gordon plantea un patrón exclusivamente para valorar la actividad física, Patrón 4: Actividad/Ejercicio, comparable a la necesidad 4 de Henderson: Moverse y mantener una postura adecuada. Punto que considero clave a la hora de valorar el estilo de vida de un deportista de alto rendimiento, ya que el primero me permite indagar sobra la cantidad de ejercicio, nada mas hacia la valoración de posibles limitaciones físicas.

Por este motivo considero que, utilizar los patrones funcionales de Gordon, enriquece mas los posibles resultados de este trabajo.

A su vez, la forma en la que M. Gordon entiende los patrones funcionales, guarda estrecha relación con el concepto de "estilo de vida", que es otro de los pilares básicos de este estudio.

\section{¿Qué relación existe entre el estilo de vida de una persona y sus patrones funcionales de salud?}

Los patrones funcionales son, de acuerdo con Álvarez Suarez (2010) una configuración de comportamientos mas o menos comunes a todas las personas, que contribuyen a su salud, calidad de vida y al logro de su potencial humano; que se dan de manera secuencial a lo largo del tiempo y proporcionan un marco para la valoración con independencia de la edad, el nivel de cuidados o la patología.

Que junto a lo que entendemos por estilo de vida: conjunto de patrones conductuales individuales (WHO, 1986) que poseen repercusiones en la salud de las personas haciendo referencia a la manera de vivir, a una serie de actividades, rutinas cotidianas o hábitos (Guerrero, L. R. 2010) podemos extraer que un estilo de vida es, básicamente, la manera que la persona tiene de gestionar los patrones funcionales de los que nos habla Gordon. Otra manera de explicar esta estrecha relación, sería diciendo que el conjunto de los 11 patrones funcionales de Gordon, componen el estilo de vida de una persona. el tipo de ejercicio, la intensidad..., mientras que la necesidad de Henderson va encami-

\begin{tabular}{|c|c|}
\hline Patrones Funcionales & Estilo de Vida \\
\hline Configuración de comportamientos & Conjunto de patrones conductuales \\
\hline Comunes en todas las personas & Particular de cada individuo (WHO) \\
\hline Contribuyen a la Salud & Posee repercusiones en la Salud \\
\hline
\end{tabular}


Podemos entender, por lo tanto, que la valoración de los patrones funcionales de Marjory Gordon es una forma de analizar el estilo de vida concreto de una persona, parte por parte

\section{JUSTIFICACIÓN}

Este trabajo nace con la prespectiva de que la Ciencia de la Enfermería, no solo se centra en la persona patológica, sinó que abarca también los estados de buena salud. Cómo ya lo definió Henderson, V. (1977) en su libro Principios Básicos de Enfermería la enfermería es "la asistencia al individuo enfermo o sano en la ejecución de aquellas actividades que favorecen su salud o la recuperación de la misma...tareas que él podría resolver sin ayuda si tuviera las energías, fuerza de voluntad y conocimientos necesarios". De este modo, la actuación de enfermería, puede contribuir a un individuo sano (como puede ser un deportista de alto rendimiento) a mantener, favorecer y mejorar su estado de salud. Pero para ello, primero hay que conocer cómo es el estado de salud de las personas con este particular estilo de vida, que se podría mejorar de él y que problemas de salud pueden venir derivados del mismo.

A su vez, durante la realización de este trabajo, he podido observar que la investigación por parte de enfermería dirigida al campo del deporte de alto rendimiento es prácticamente nula y por lo tanto necesária para la ampliación de conocimientos de la comunidad enfermera y científica en general.

\section{OBJETIVOS E HIPÓTESIS}

Dada la gran cantidad de puntos de vista diferentes desde los que se puede abordar el deporte de alto rendimiento como factor protector de salud o factor de riesgo, con este estudio nos planteamos los siguientes objetivos:
- Conocer la relación que guarda el deporte de alto rendimiento con cada uno de los patrones funcionales de salud de la persona.

- Obtener una visión más holística de este estilo de vida que nos ayude a entender y diriguir los cuidados de enfermería que un deportista de alto rendimiento pueda precisar.

También nos hemos planteado las siguientes hipótesis:

- No se puede afirmar de forma generalizada que el deporte de alto rendimiento sea promotor de salud o factor de riesgo, ya que este estilo de vida puede afectar tanto positivamente como negativamente a los diferentes patrones funcionales de salud.

- El hecho de ser deportista de alto rendimiento, no tiene porque afectar a todos los patrones funcionales de salud.

- Un patrón funcional concreto puede que se vea favorecido y perjudicado al mismo tiempo por la practica de deporte de alto rendimiento.

\section{METODOLOGÍA}

Este trabajo es un estudio de caso que utiliza metodología cualitativa y como técnicas principales, la entrevista en profundidad y una tabla semanal (del 28 de enero al 3 de febrero de 2013) que recoge información relativa a la alimentación y la actividad física en periodo de competición.

La elección de esta metodología se ha realizado teniendo en cuenta los siguientes aspectos:

Desde la ciencia social que representa la enfermería, la perspectiva cualitativa, nos permite estudiar los fenómenos en su contexto y se les puede encontrar el sentido o la interpretación partiendo del significado que las personas les concedemos. Nos centramos por lo tan- 


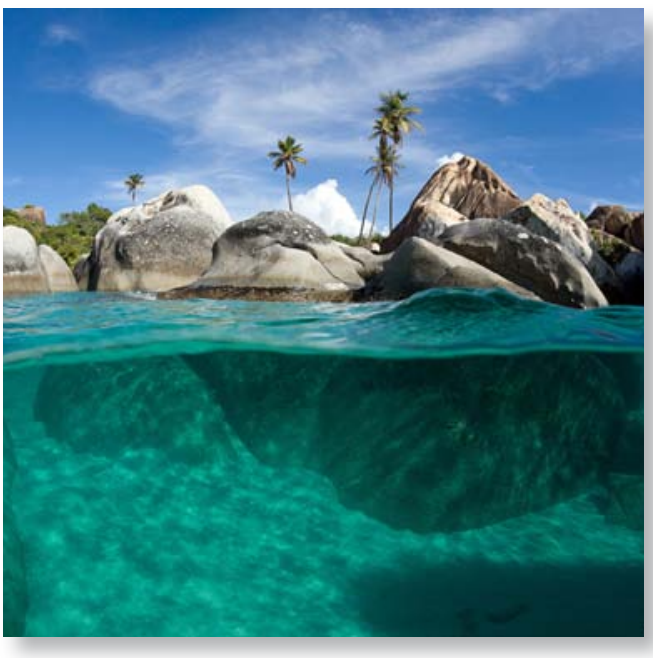

to en la multidimensionalidad de la realidad social (Calderón, C. 2002). Esto es interesante a la hora de valorar el estilo de vida propio de una persona, dado que esta perspectiva proporciona una visión holística (Burns, N. 2004) del modo de vida del informante. Hecho que enriquece la información que obtenemos y el análisis de la misma.

La investigación cualitativa, ayuda a responder preguntas surgidas desde el significado de la experiencia de vida y de la comprensión de sentimientos de la persona (Orellana. A. 2007). Es fundamental para la valoración de los patrones funcionales tener en cuenta la subjetividad con la que la persona comprende e interpreta su estilo de vida. Esta metodología, nos ayuda a explorar en profundidad, la riqueza y la complejidad inherente a un fenómeno, como es la valoración de los patrones de Gordon. Por lo tanto, la investigación cualitativa es útil para entender las experiencias humanas (Burns, N. 2004).

Para llevar a cabo este trabajo, ha sido necesaria la colaboración de un informante. Los principales criterios de selección, han sido que cumpliese con los requisitos propios del estilo de vida que pretende analizarse, es decir, que sea una persona joven y a su vez realice una modalidad deportiva de alto rendimiento. (Entendiendo como joven el periodo de edad de entre 20-24 años (OMS, 1986) y como deporte de alto rendimiento la actividad física que obedece normas y sus objetivos son la competencia, el máximo rendimiento y el espectáculo (Ortiz U, 2001) que se realiza de forma organizada y consciente y está diseñado para aumentar el nivel competitivo y el rendimiento atlético, es un proceso organizado, dirigido, orientado, sistemático, planificado y controlado sobre bases metodológicas (Gómez, O. 2002).

Para la localización del informante he decidido buscar en las universidades, ya que es un lugar donde confluyen gran cantidad de jóvenes y la probabilidad de encontrar a alguno que se dedique al deporte de alto rendimiento es mas elevada. Para ello, y por motivos de cercanía que facilitarían la realización de las entrevistas, decidí empezar a buscar en mi propia universidad. $Y$ en caso de no encontrar ningún posible informante, ir expandiendo el perímetro hasta hallar una persona que cumpla los criterios de selección y esté dispuesta a colaborar. En mi propia universidad encontré una chica que, tras explicarle en persona en que consistía el trabajo y de que forma podría ayudarme, aceptó ser mi informante a través de un consentimiento verbal.

Se trata de una chica de 21 años que practica patinaje artístico en su modalidad grupal. Nació en Lleida, pero se trasladó a vivir a Tarragona hace 4 años por motivos universitarios. Lleva patinando desde 1995. Al venir a vivir a Tarragona, en el 2009, se incorporó al equipo de patinaje Reus Deportiu, con el que ha cosechado grandes éxitos profesionales. Entre el palmarés que avala la elección del informante, encontramos: un título de subcampeona del mundo en el Mundial de Alemania en 2009. 
Una medalla de bronce en el Mundial de Brasil 2011 y una medalla de oro en el Mundial de Nueva Zelanda en el año 2012. Además de títulos como Campeona de España en 2012 y subcampeona de Europa ese mismo año.

Este estudio ha sido realizado durante el transcurso de noviembre de 2012 a junio de 2013 en la provincia de Tarragona.

Para la consecución de los objetivos, se ha llevado a cabo una entrevista en profundidad dirigida hacia una valoración de los patrones funcionales de Marjory Gordon.

Para el análisis de datos, he utilizado el Manual de Valoración de Patrones Funcionales (2010) realizado por un equipo del Área Sanitária V de Gijón (Asturias) formado por José Luis Álvarez Suarez, Fernanda del Castillo Arévalo, Delia Fernández Fidalgo y Montserrat Muñoz Meléndez.

A la hora de realizar la entrevista he tenido en cuenta que valora y cómo se valora cada patrón, dirigiendo siempre las preguntas hacia la posible relación que pueda guardar cada ítem de cada patrón funcional con el estilo de vida de un deportista de alto rendimiento.

Para extraer resultados, he elaborado una tabla para cada patrón funcional en la que constan todas las acciones, signos, síntomas y estados (ítems) que, según el Manual de Valoración de los Patrones funcionales, alteran el patrón en concreto. De cada aspecto que deteriora un patrón, he redactado lo opuesto de tal modo que obtengo el mismo ítem, pero esta vez beneficia al patrón en si. Un ejemplo de esto sería:

En la guía, en el Patrón Funcional 1(Percepción de la Salud) expone como ítem que altera negativamente el patrón funcional lo siguiente: "Bebe en exceso, fuma y/o consume drogas". De este ítem he extraído el que beneficia al patrón funcional que sería: "No bebe en exceso, no fuma ni consume drogas". Este proceso se ha seguido con todos los ítems que propone la guía para cada patrón funcional.

Una vez realizada la entrevista y las tablas de los ítems (factores protectores o conductas de riesgo) de cada patrón, se procede a la valoración de enfermería en la que se analizan todos los patrones y se escogen los ítems que encajen con el estilo de vida que el informante nos facilitó en la entrevista. Una vez marcados todos, resalto los que tienen que ver con el hecho que el informante practique deporte de alto rendimiento. De este modo obtengo como el deporte de alto rendimiento afecta a cada patrón, justificando cada resultado confrontando cada respuesta del informante que nos lleve a ese resultado con las aclaraciones que nos propone el Manual de Valoración de los Patrones Funcionales para cada ítem.

\section{RESULTADOS Y DISCUSIÓN}

Para presentar los resultados voy a exponer los patrones por orden, uno a uno.

Patrón funcional 1; Percepción/Manejo de salud. En este patrón encontramos dos ítems positivos afectados por el deporte de alto rendimiento, que son, el hecho que tiene un adecuado cuidado de su salud: en este ítem se puede englobar la valoración general de todos los patrones funcionales, que vienen derivados de la gestión de su estilo de vida (que gira alrededor de su condición de deportista de alto rendimiento), que cómo bien puede apreciarse en las tablas de valoración (anexo), nos muestra un buen manejo de su salud. Y segundo, que no bebe en exceso, no fuma y no consume drogas: comenta que no fuma ni consume drogas, y que consume alcohol de manera muy esporádica cuando sale de fiesta (si su ritmo de vida se lo permite). En una semana de 
consumo podríamos hablar de $40 \mathrm{gr} /$ semana (siendo una vez al mes) Y el consumo excesivo en mujeres se considera a partir de los 168gr/ semanales. En la entrevista comenta que "está claro que el estilo de vida influye ya que el hecho de hacer deporte y juntarte con personas que tienen hábitos de vida saludables te ayuda a mantenerte lejos de ciertos estímulos negativos"

Patrón funcional 2, Nutricional/Metabólico: en este patrón funcional vemos que el deporte de alto rendimiento afecta de forma directa a un ítem positivo y es que tiene una nutrición equilibrada: Tal cómo se observa en el planning horario semanal (anexos), hay una buena repartición de los nutrientes que consume, siendo una dieta equilibrada, rica en proteínas y baja en grasas. En la entrevista comenta que su condición de deportista de alto rendimiento la condiciona a llevar este tipo de dieta para "mantener un poco el peso normal para aguantar lo que te exige tu deporte. Nadie te obliga, pero de alguna manera te cuidas para eso, para facilitar el aguantar y el practicar tu deporte".

Patrón funcional 3; Eliminación: en este patrón funcional vemos como el deporte de alto rendimiento afecta al mismo ítem de forma positiva y negativa. Presenta un tránsito intestinal regular y sin problemas. Acostumbra a ir al baño una o dos veces al día. Comenta que "hacer ejercicio mejora la eliminación, porque, quieras o no, es algo que te mantiene continuamente en movimiento y yo creo que lo facilita". Pero a su vez, existe estreñimiento y diarrea. En periodos de competiciones y debido a las rivalidades y autoexigencias propias del deporte de alto rendimiento, se ve alterado su ritmo intestinal debido a nervios y estrés. Y esto deriva en "defecaciones muy fáciles o estreñimientos espectaculares". Aunque son situaciones puntuales que una vez superada la competición dejan paso al ritmo normal.

Patrón funcional 4; Actividad/Ejercicio: Dentro de este patrón, se ven alterados 3 ítems, uno positivo y dos negativos. Como ítems negativos encontramos el riesgo de accidentes/ lesiones. En la entrevista comenta una rotura de peroné en 2007 durante una competición además de "como todo deportista, lo típico, roturas fibrilares, sobretodo en piernas, contracturas, y alguna cosa más sin importancia”. Por lo tanto, de esta práctica deportiva se deriva un importante riesgo de lesiones. Y por otro lado, un cansancio físico relacionado con exceso de actividad. Esto se ve reflejado en la gran cantidad de horas dedicada al deporte "mas horas no le puedo dedicar, porque si le dedico mas horas o quito las horas de comer o quito las horas de dormir". Acompañado de consumo de suplementos vitamínicos para poder aguantar el ritmo que requiere este tipo de práctica deportiva. El ítem positivo hace referencia a la actividad lúdica y el entretenimiento. No manifiesta aburrimiento, comenta que el hecho de ser deportista de alto rendimiento la ha condicionado a buscar una vida activa y en su tiempo libre le gusta probar deportes nuevos, quedar con amigos, etc.

Patrón funcional 5; Sueño/descanso: El deporte de alto rendimiento afecta directamente dos ítems negativos de este patrón. Por un lado verbaliza dificultad para conciliar el sueño. Comenta en la entrevista "Estoy muy cansada, pero no tengo sueño, porque llegas a casa con el pensamiento acelerado de todo el deporte que acabas de hacer. Entonces es eso, que estás muy cansada, con muchas ganas de dormir pero me cuesta conciliarlo". Y por otro, refiere 
cansancio relacionado con falta de descanso. Comenta "No recupero las horas de sueño, a veces me gustaría dormir mas y poder estar mas horas durmiendo" aunque comenta dormir unas 7-8 horas diarias, lo recomendado en adultos, la carga física que comporta ser deportista de alto rendimiento puede ser el factor que haga que esas horas sean insuficientes.

\section{Patrón funcional 7; Autopercepción/Au-} toconcepto: Un ítem negativo de este patrón se ve afectado directamente por la práctica de deporte de alto rendimiento y es que le preocupa su imagen corporal y los cambios de esta. Verbaliza que "el hecho de estar en la alta competición y querer hacerlo todo perfecto, te hace buscar también la perfección en ti”. Como comentaba J. Alonso (2006), en los deportes femeninos tales cuales la gimnasia rítmica o el patinaje artístico el componente de la imagen física tiene mucho mas peso que en otros deportes llegando a producir obsesiones y TCA, aunque este no sea el caso de nuestra informante, si que se le da cierta importancia a la imagen física.

Patrón funcional 8; Rol/Relaciones: Cubre correctamente todos los ítems de este rol, pero debido al su vida como deportista de alto rendimiento expresa un ítem negativo; por su ritmo de vida, se siente a veces separada del resto de amigos y compañeros que no practican como ella deporte de alto rendimiento.

Responde en la entrevista a la pregunta $¿$ Crees que tu estilo de vida ha podido influir en tus relaciones sociales? "Si, si, totalmente, he ido a Lleida a ver a mis amigos y es como a veces me da la sensación de que ellos están en un mundo y yo estoy apartada [...] Con la gente de patinaje o que hace deporte, no. Pero con la gente que no hace deporte si que ves este distan- ciamiento." Y esto viene dado a que, a muchas de las actividades que realizan los compañeros de la edad del informante como salir de fiesta, quedar con cierta regularidad... mi informante, por su ritmo de vida y su condición de deportista de alto rendimiento no puede acudir.

Patrón funcional 10, Adaptación/Tolerancia al estrés: Dos ítems positivos de este patrón se ven afectados directamente por el deporte de alto rendimiento. Afronta cambios de manera efectiva. Comenta que a la hora de afrontar problemas "el estilo de vida si que influye, en cualquier modalidad deportiva pueden surgir muchos cambios, sin ir mas lejos, como la propia coreografía, y te vas adaptando." Esta actitud de adaptabilidad aprendida en el mundo del deporte, es aplicada en otros ámbitos de su vida. Y no recurre al uso de sustancias para superar problemas. Comenta que el deporte, el patinaje, es una vía de escape "me pongo los patines $y$ entro en otro mundo.... Me olvido de todo [...] son formas... por eso no he recurrido a medicamentos. Te vas a correr y mañana será otro día." En este caso, el deporte es una manera de canalizar los problemas de manera natural y sin perjudicar a la salud.

Podemos resaltar como factor protector, que el hecho de ser deportista de alto rendimiento ha conllevado al informante a desarrollar un patrón de hábitos saludables como son la alimentación, el ejercicio físico regular y han ayudado a alejarse de estímulos nocivos como drogas o tabaco. Este dato está reflejado en las conclusiones del estudio de Alejandro Lucía (2006).

Por el contrario, podemos entender como principales factores de riesgo el hecho que la práctica de deporte (alto rendimiento o no (Amato, R. 2000) conlleva riesgo de lesiones.

Pero al tratarse de deporte de alto rendi- 
miento, la persona se ve expuesta una gran cantidad de horas a ese riesgo, y por lo tanto, es relativamente más fácil que sufra algún tipo de lesión a lo largo de su trayectoria.

También, la gran cantidad de horas de dedicación que requiere el deporte de alto rendimiento conlleva dos puntos de relevante interés, que son: el hecho de la falta de tiempo para poder realizar otras actividades de carácter social y el cansancio físico que conlleva un ritmo de vida tan intenso y prolongado en el tiempo. Este segundo aspecto negativo es el responsable del burnout (Marquez, S, 2005) que, durante la entrevista, mi informante comentó que hizo que algunas de sus compañeras de equipo decidiesen abandonar el deporte a ese nivel. Por lo tanto es un factor importante a tener en cuenta a la hora de tratar con deportistas de alto rendimiento.

Aunque a grosso modo, de la valoración realizada a mi informante, se puedan extrapolar varios resultados a otros deportistas de alto rendimiento, cómo pueden ser la conducta relacionada con hábitos saludables o la gran dedicación y esfuerzo que pueden conllevar a un cansancio físico y psicológico crónicos, hay que tener en cuenta que cada persona puede gestionar su estilo de vida de diferente manera y hay variables, como puede ser el grado de dedicación o el tipo de deporte que se practique, que pueden hacer variar los resultados, de tal manera que puedan surgir nuevos factores protectores o nuevas conductas de riesgo en personas que compartan un estilo de vida similar siendo jóvenes deportistas de alto rendimiento.

\section{CONCLUSIONES}

Por lo general, el estilo de vida del informante se puede valorar positivamente como puede verse en las tablas de valoración (ane-



xos) que muestran 52 ítems positivos frente a 9 ítems negativos. En lo que refiere al hecho de practicar deporte de alto rendimiento, vemos que esta modalidad deportiva produce alteraciones directas e indirectas a muchos ítems de varios patrones funcionales de salud. De los 52 ítems positivos, 7 son consecuencia directa de la práctica de deporte de alto rendimiento. Y de los 9 negativos, 7 vienen derivados de este tipo de práctica deportiva.

Los patrones alterados (tanto de manera positiva como negativa) por la condición de deportista de alto rendimiento son el 1, 2, 3, $4,5,7,8$ y 10 .

\section{AGRADECIMIENTOS}

Agradezco a Lina Casadó Marín, doctora del Departamento de Enfermería de la Universitat Rovira i Virgili su apoyo y tutorización durante el desarrollo de este trabajo.

$\mathrm{Y}$ agradecer también a mi informante su voluntariedad y paciencia.

\section{BIBLIOGRAFÍA}

- Akandere, M. y Tekin, A. (2005). Efectos del ejercicio físico sobre la ansiedad. PubliCE Standard; Pid:478. Alonso, J (2006) Trastornos de la conducta alimentaria y deporte. Trastornos de conducta alimentaria 4. Centro Andaluz de Medicina del Deporte 368-385 
- Álvarez Suárez, J. L. del Castillo Arévalo, F. Fernández Fidalgo, D. Muñoz Meléndez, M. (2010) Manual de Valoración de Patrones Funcionales, Dirección de Enfermería de Atención Primaria Área Sanitaria V - Gijón.

- Amato, R. (2000) Deporte competitivo infanto juvenil: ¿promoción de la salud o situación de riesgo? Adolescencia Latinoamericana., vol.2, no.1, p.09-15

- Arruza, J. A. Arribas, S. (et.al.) (2011) Percepción de competencia, estado de ánimo y tolerancia al estrés en jóvenes deportistas de alto rendimiento Universidad del país Vasco, vol. 27, n² 2, p.536-543

- Barrios-Cisnero, H. A. (2007). Estilo de Vida Saludable y Espiritualidad. Monografía s/p. Mérida, Venezuela.

- De Gracia M. y Marcó M. (2000). Efectos psicológicos de la actividad física en personas mayores. Psicothema. $12,2,285-292$.

- Durand, M. (1988), El niño y el deporte, Barcelona, Paidós Ibérica S.A y Centro de Publicaciones del Ministerio de Educación y Ciencia, pp:.113-114.

- Freudenberger, H.J. y Richelson, G. (1981). Burnout: How to beat the high cost of success. New York: Bantam Books.

- García Fernando, M. (1990), Aspectos sociológicos del deporte. Una reflexión sociológica. Alianza Editorial, Madrid, pp:148-155,202, 204.

- García Suso, A, Francisco del Rey C, Palazuelos Puerta P, de Bustos M. L. (2004) El modelo conceptual de Virginia Henderson y la etapa de Valoración del Proceso de Atención de Enfermería. Educare21; No 9.

- Guerrero Montoya L.R. y León Salazar A.R. (2010) Estilo de vida e saúde. Artículos arbitrados, No 48, Enero-Junio, p. 13-19

- Gómez Acosta, Oscar Fundamentos Generales de la Caracterización y Organización del Deporte de Alto Rendimiento Cuba 2002

- Henderson V. (1971) Principios Básicos de los Cuidados de Enfermería. Ginebra: Consejo internacional de enfermeras.

- Holmes, D.S. (1993). Aerobic fitness and the response to psychological stress. En P. Seraganian (Ed.), Exercise psychology. The influence on physical exercise on psychological process (pp. 39-63). Nueva York: John Wiley
- Jenkins, David. (2005). Mejoremos la salud a todas las edades. Un manual para el cambio de comportamiento. Organización Panamericana de la Salud. Washington, D.C.

- Jiménez, M., Martínez, P., Miró, E. y Sánchez, A. (2008). Bienestar psicológico y hábitos saludables ¿están asociados a la práctica del ejercicio físico. Journal of Clinical and Health Psychology, 8, 185-202.

- Jürgens, I. (2006) Práctica deportiva y percepción de calidad de vida. Revista Internacional de Medicina y Ciencias de la Actividad Física y el Deporte vol. 6 (22) pp. $62-74$

- Lawlor, D.A. y Hopker, S.W. (2001). The effectiveness of exercise as an intervention in the management of depression: Systematic review and meta-regression analysis of randomized controlled trials. British Medical Journal, 322, 763-767.

- Lucia A, (et. Al.) (2006) ACTN3 genotype in professional endurance cyclists. Int J Sports Med 2006; 27: 880 - 884

- Marquez, S. (2005) Estrategias de afrontamiento del estrés en el ámbito deportivo: fundamentos teóricos e instrumentos de evaluación. International Journal of Clinical and Health Psychology. Vol.6, No2, pp. 359-378.

- Muñoz Guillén, J. A. (2001) Definición de Deporte, Quincena 2, Mayo de 2001. Avaiable http: www.pdf-esmanual.com/books downloaded 10 de diciembre de 2012

- Ruiz, J. (2010) Strenuous endurance exercise improves life expectancy: it's in our genes British Journal of Sports Med 2011;45:3 159-161 Published Online First: 27 September

- Souto, A. y Fariña, F. (2009, octubre). Práctica deportiva en la tercera edad y su efecto en la salud física y psicocognitiva. XI Jornadas Nacionales y I Congreso Internacional de Fisioterapia en la Actividad Física y del Deporte. Madrid.

- WHO (1986). Life styles and Health. Rv.Social Science and Medicine, 22 (2): 117-124. 